\title{
Public health aspects of tuberculosis
}

\section{${ }^{1} \mathrm{O}$ Blatchford, ${ }^{2} \mathrm{JC}$ Cameron}

${ }^{1}$ Consultant in Public Health Medicine; ${ }^{2}$ Strategic Lead Vaccine Preventable Diseases (Respiratory Bacteria), Health Protection Scotland, Glasgow, UK

\begin{abstract}
This article covers public health aspects of the investigation and management of people who are infected with tuberculosis (TB). It contains a brief overview of the recent epidemiology of TB in Scotland, focusing on changes in Scottish TB incidence and describing some epidemiological associations. We then describe the initial public health assessment of those with suspected TB and responses that should be initiated. It does not address issues relating to the clinical treatment of patients with TB.
\end{abstract}

Correspondence to $O$ Blatchford, NHS Health Protection Scotland, Meridian Court (4th floor), 5 Cadogan Street, Glasgow G2 6QE, UK

tel. +44 (0) I4I 2822943 e-mail oliver.blatchford@nhs.net

KEYWORDS Tuberculosis, epidemiology, public health, Scotland

DECLARATION OF INTERESTS No conflicts of interest declared.

\section{OVERVIEW}

Tuberculosis (TB) is usually caused by infection with Mycobacterium tuberculosis. There are four other species of mycobacteria which rarely cause human TB infections. These are M. bovis, M. africanum, M. canetti and M. microti. Together with $M$. tuberculosis, they are collectively referred to as the 'TB complex'.

Although TB infection commonly involves the respiratory tract, it frequently infects other anatomical sites. If the disease process affects the lungs, pleural cavity, mediastinal lymph nodes or larynx, it is defined as pulmonary TB. It is defined as non-pulmonary TB if other sites are infected and there is no involvement of pulmonary sites. Tuberculosis is mainly transmitted by the airborne route, although other transmission mechanisms may be seen in practice, for example food borne transmission leading to enteric TB disease or transmission by direct exposure to fluid draining from a tuberculous abscess. Haematogenous spread from a primary respiratory infection may also result in infection in a remote non-pulmonary site.

Most people who are infected with $M$. tuberculosis are likely to recover from the initial infection; however, approximately $5 \%$ of those infected will develop active TB disease. This is usually a slowly progressive condition and is likely to be fatal if untreated. In about half of those who recover from the initial TB infection, the mycobacteria continue to survive in a latent state, remaining viable but dormant for many years. Such a latent infection may subsequently be reactivated,

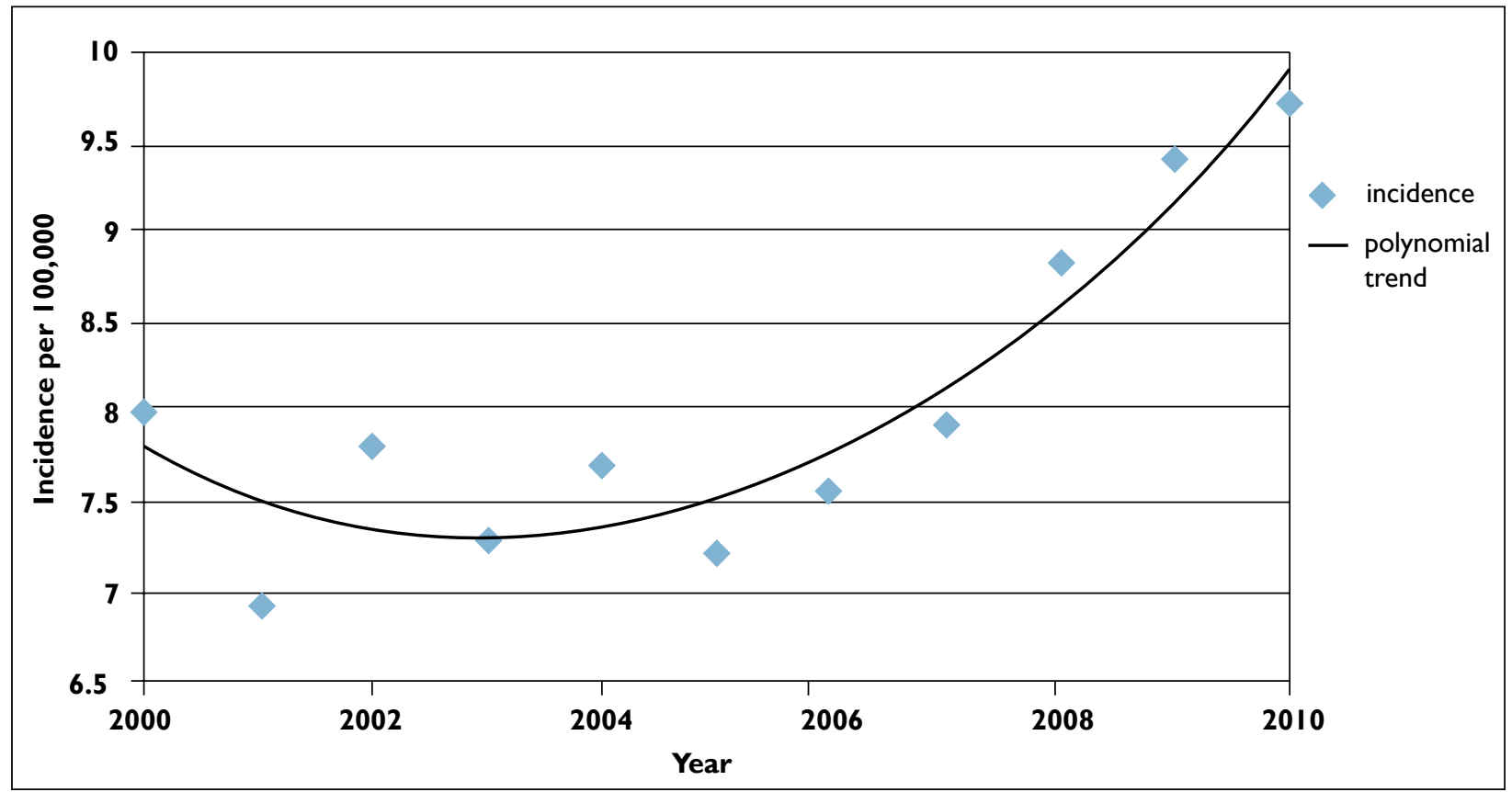

FIGURE I Tuberculosis incidence in Scotland (rate per 100,000 population) 2000-10, with polynomial trend line. 
resulting in a recurrent TB infection. Reactivation of latent TB is more common among the elderly and the immunosuppressed, including those with human immunodeficiency virus (HIV) infections.

The incidence of TB in Scotland and the rest of the UK has been rising during the past decade. This has also been seen elsewhere in Europe, where changes in patterns of travel and migration have led to TB becoming a public health problem in many other countries. Scotland has also been affected by rising numbers of patients with TB infections, with 506 TB notifications in 2010. This represented an incidence of 9.7 TB infections per 100,000 in the population. Compared with 2009 , this was an increase of $4.1 \%$ in the number of notified infections and $3.2 \%$ in the incidence. This increase has been seen every year since 2005 (Figure I). However, Scotland has had a lower incidence than the UK taken as a whole (I3.6 per I00,000) and in particular, lower than London (42 per 100,000). For many years, the largest numbers of notified infections in Scotland have been from the central belt, from the Greater Glasgow and Clyde, Lothian and Lanarkshire NHS Boards areas, as well as the Grampian area.

Tuberculosis has a substantial associated mortality. In $2010,10 \%$ of Scottish cases died within one year of their diagnosis (42 cases). Of these, $69 \%$ died before their treatment had been completed, while $21 \%$ were postmortem diagnoses (having never commenced treatment).

\section{WHO IS LIKELY TO GET TB IN SCOTLAND}

In 2010, more than half of notified Scottish TB infections occurred in males, who were more commonly affected than women in all age groups (except for children under five years and adults over 75 years; however these groups accounted for relatively few patients). The overall highest TB incidence was amongst those aged 25-34 years (19.2 per 100,000, representing $25.5 \%$ of the total number of cases in Scotland) (Figure 2). It should be noted that the age distribution of TB patients is associated with their place of birth, as discussed below.

It is important to note that the incidence of TB among children under five years old has increased from 2.8 in 2009 to 3.4 notified infections per 100,000 in 2010 . Infections in this age group are a widely accepted marker of continuing transmission of TB within a population as they are due to recent infections rather than to reactivation of latent TB acquired during previous episodes of infection. This suggests that TB transmission is continuing in Scotland.

Several patient risk factors have been reported as being associated with increased risk of TB in Scotland. These include:

- Alcohol misuse

- Immunosuppression (including HIV infection)

- Working in healthcare

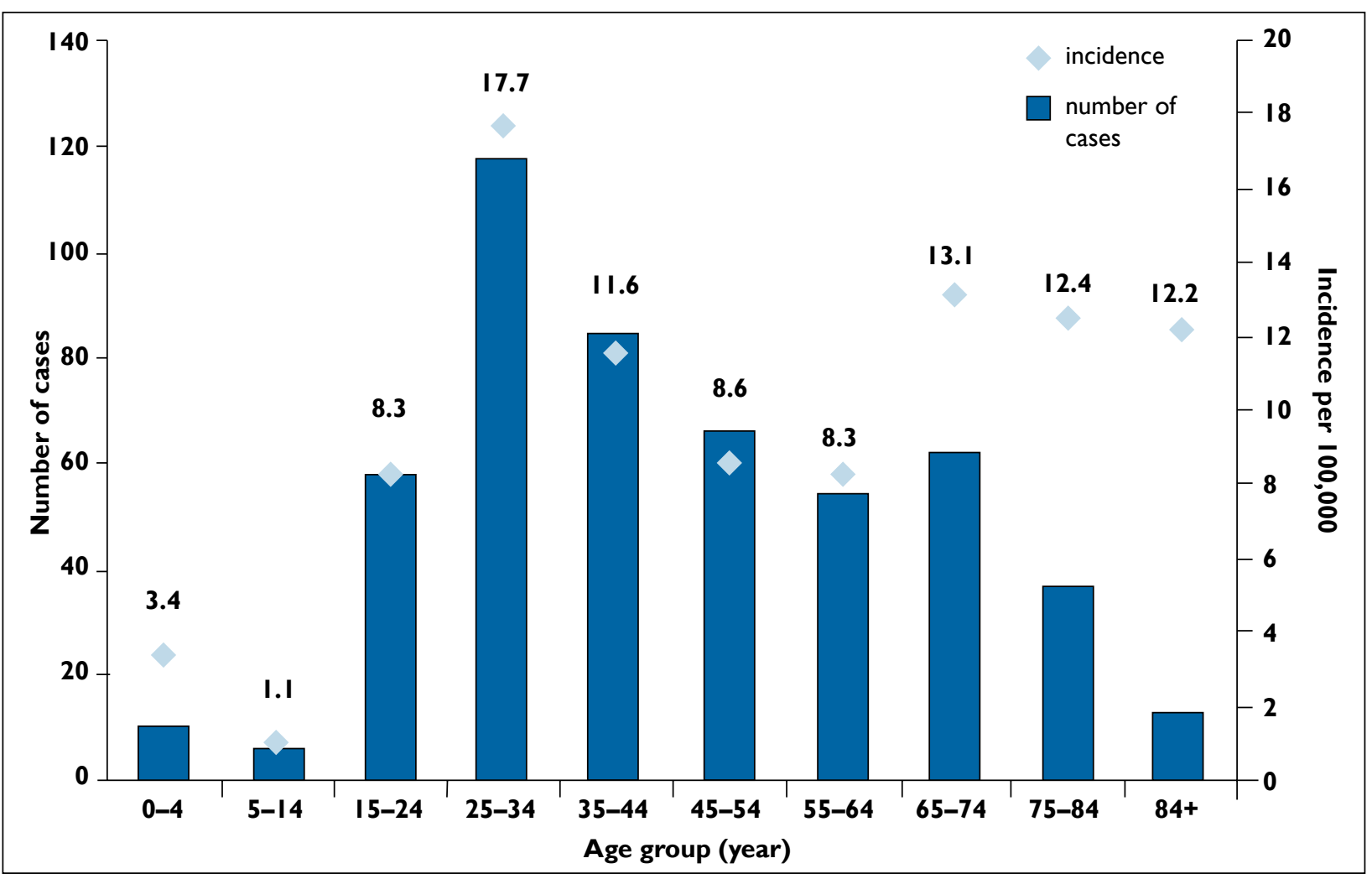

FIGURE 2 Number of tuberculosis cases in Scotland and rate per 100,000 population by age group (2010). 


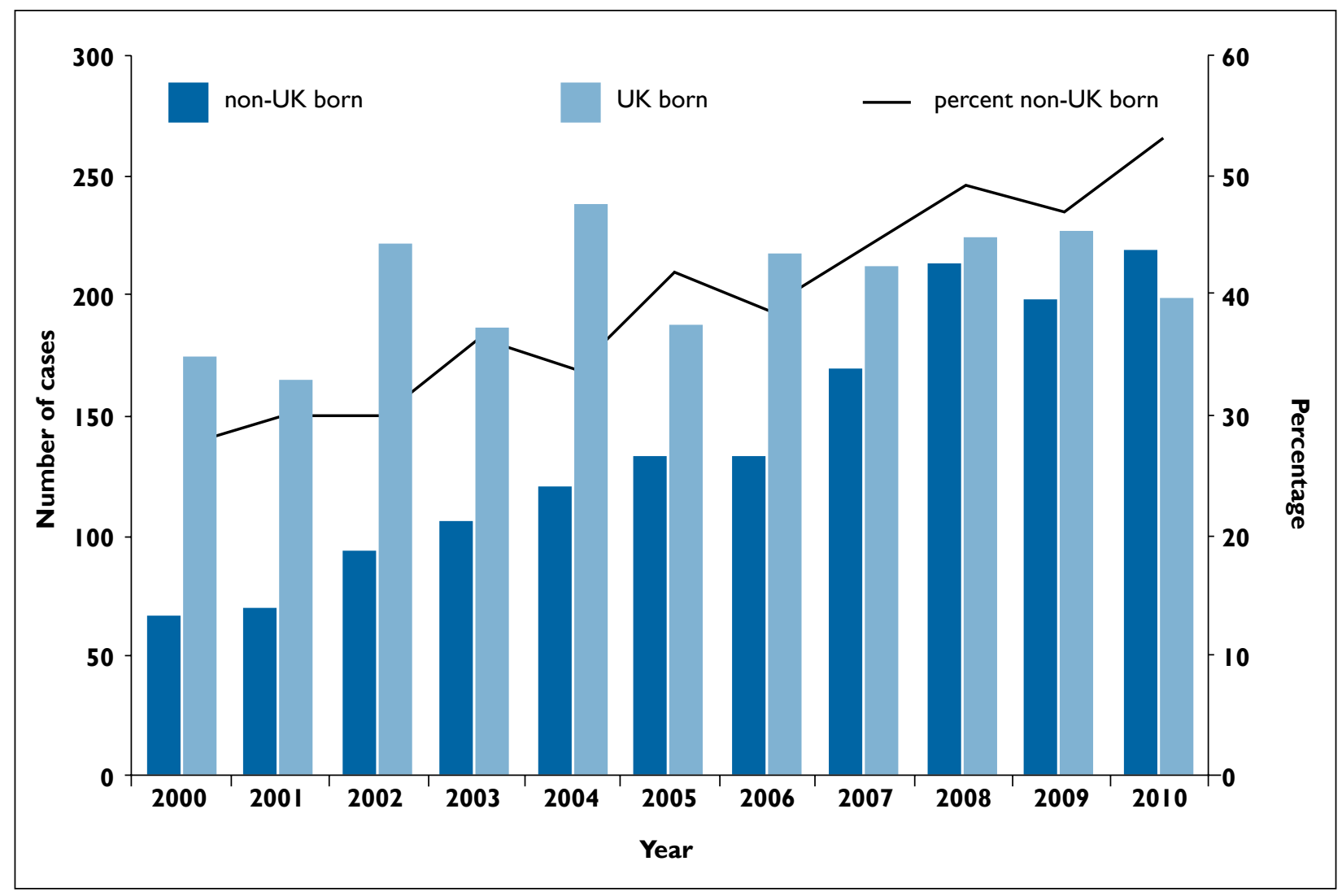

FIGURE 3 Proportion of tuberculosis cases in Scotland, born outside the UK (2000-10).

- Being a refugee

- Residence in a residential or corrective institution

- Homelessness

The place of birth was recorded for $86.2 \%$ of patients in 2010 . Of these, $52.5 \%(217 / 413)$ were born outside the UK. This reflects a rising trend in both the numbers and proportions of notified TB patients who were not born in the UK. Figure 3 shows that both have risen substantially since 2000 . There was also a significant difference in the ages of notified TB patients depending on where they were born (Figure 4). The average age of Scottish TB patients notified in 2010 who were born in the UK was $5 \mathrm{I}$ years, while those born outside the UK had an average age of 36 years. For these notified patients whose country of birth was reported, Pakistan was the most commonly recorded country, with 62 notifications representing $28.6 \%$ of those born abroad, followed by India $(57 ; 26.3 \%)$ and Nigeria $(14 ; 6.5 \%)$ in 2010 (Table I). DNA fingerprinting studies have suggested that most of these infections arise from reactivation of latent TB infections which were acquired outside the UK.

Although there has been a rise in the proportion of notified TB infections among those born abroad, it is still important to note that most TB infections in Scotland are found in ethnic Caucasians (Table 2), reflecting the demography of Scotland and the fact that TB remains a problem for the indigenous Scottish population. It should also be noted that $71.7 \%$ of Scottish TB patients born outside the UK had entered the UK two or more

TABLE I Countries of birth for non-UK born tuberculosis cases in Scotland (2010)

\begin{tabular}{|l|c|c|}
\hline Country of birth & $\begin{array}{c}\text { Number of } \\
\text { cases }\end{array}$ & $\begin{array}{c}\text { Percentage of } \\
\text { cases }\end{array}$ \\
\hline Pakistan & 62 & $28.6 \%$ \\
\hline India & 57 & $26.3 \%$ \\
\hline Nigeria & 14 & $6.5 \%$ \\
\hline Bangladesh & 7 & $3.2 \%$ \\
\hline China & 7 & $3.2 \%$ \\
\hline Romania & 6 & $2.8 \%$ \\
\hline Somalia & 6 & $2.8 \%$ \\
\hline Zimbabwe & 5 & $2.3 \%$ \\
\hline Nepal & 4 & $1.8 \%$ \\
\hline Phillipines & 4 & $1.8 \%$ \\
\hline Gambia & 3 & $1.4 \%$ \\
\hline Poland & 3 & $1.4 \%$ \\
\hline Thailand & 3 & $1.4 \%$ \\
\hline Others (each $<1 \%)$ & 35 & $16.2 \%$ \\
\hline Unknown & 1 & $0.5 \%$ \\
\hline Total & 217 & $100 \%$ \\
\hline
\end{tabular}




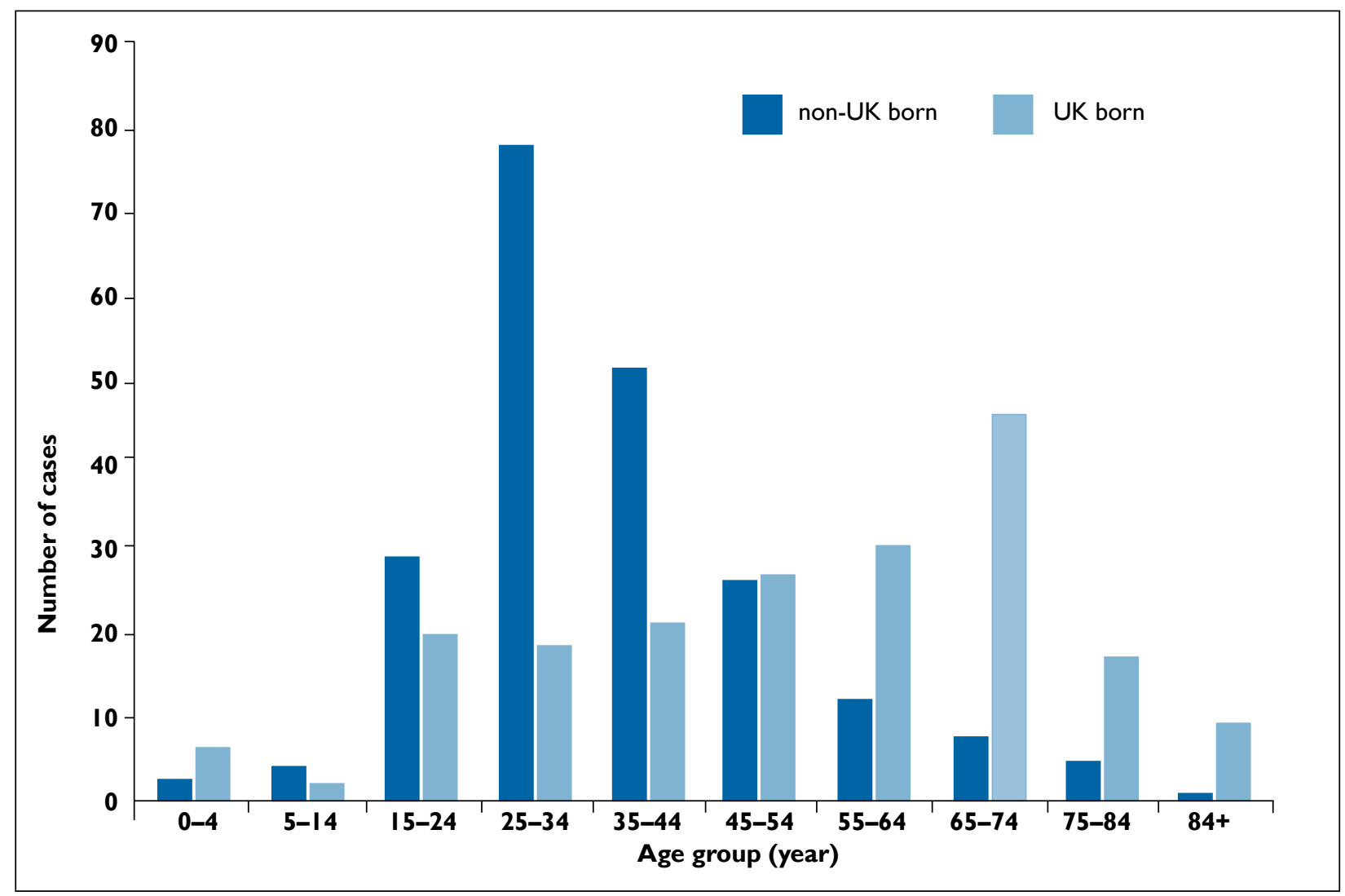

FIGURE 4 Tuberculosis cases by age group and place of birth in Scotland (2010).

years before they were diagnosed with TB $(42.3 \%$ had entered five or more years earlier). This suggests that screening for active TB at ports of entry to the UK is unlikely to be an effective control measure.

The past decade has seen a relative increase in the proportion of non-pulmonary TB cases, compared with pulmonary TB (Figure 5). Similar changes have been seen across the UK.

\section{PREVENTION OF TB}

Public health activity to reduce the incidence of TB in the UK has been directed around reducing risks of acquiring infection from other people who are already infected with the disease. This has centred on active case finding followed by treatment of those found to be infected with TB. In the 1950s and 1960s, a mass miniature radiographic screening programme was used to screen the population for TB. Mobile X-ray vans were deployed in community settings as well as workplaces to identify TB patients, ideally while they were still asymptomatic in early stages of the illness. The World Health Organization recommended that most countries should discontinue these programmes in the 1970s as the incidence of TB had declined dramatically, so the cost could no longer be justified and as the technology then in use involved exposure to significant radiation doses. There are other routes of transmission which may lead to infection. Drinking milk from cows infected with $M$. bovis may result in infection in the gastrointestinal tract. Pasteurisation of milk has largely eliminated this risk.

Primary prevention of TB has also been undertaken by immunisation in the UK since 1953. This was based on the Bacillus Calmette-Guérin vaccine (BCG), which is a live attenuated strain derived from $M$. bovis. The programme was initially targeted at children of schoolleaving age (then 14 years old), as the peak incidence of TB was in young, working-age adults.

TABLE 2 Ethnic origin of tuberculosis cases in Scotland (2010)

\begin{tabular}{|l|c|c|}
\hline Ethinic origin & $\begin{array}{c}\text { Number of } \\
\text { cases (\%) }\end{array}$ & $\begin{array}{c}\text { Of which were } \\
\text { non-UK born }\end{array}$ \\
\hline White Caucasian & $228(47.8 \%)$ & $8(3.5 \%)$ \\
\hline Pakistani & $83(17.4 \%)$ & $63(75.9 \%)$ \\
\hline Indian & $69(14.5 \%)$ & $58(84.1 \%)$ \\
\hline Black African & $46(9.6 \%)$ & $44(95.7 \%)$ \\
\hline Other/mixed & $31(6.5 \%)$ & $28(90.3 \%)$ \\
\hline Chinese & $10(2.1 \%)$ & $8(80.0 \%)$ \\
\hline Bangladeshi & $9(1.9 \%)$ & $7(77.8 \%)$ \\
\hline Black Caribbean & $\mathrm{I}(0.2 \%)$ & $\mathrm{I}(100 \%)$ \\
\hline
\end{tabular}


The schools-based BCG programme was discontinued in 2005 because the incidence of TB in the UK-born population had declined to such an extent that the use of the vaccine could not be justified for this population. Instead, the risk-based neonatal BCG programme targeted at protecting those children most at risk of exposure to TB was strengthened. It is also currently offered to the children of new entrants from countries with high TB incidence.

\section{PUBLIC HEALTH ASSESSMENT AND EARLY MANAGEMENT OF SUSPECTED TB}

The clinical diagnosis and management of active TB disease should be undertaken by TB specialists, who will usually be paediatricians or respiratory or infectious disease physicians. However, this should be done in consultation with a wider multi-disciplinary team with specialist input from other disciplines including TB nursing, public health, microbiology and pharmacy. The initial diagnosis of TB is likely to have been made in a clinical setting. The diagnosing clinician should inform the local TB nursing team as well as formally notifying the public health department. It is essential that multiple samples are sent to the microbiology service for TB microscopy and culture, preferably before any antituberculous treatment is initiated. For suspected pulmonary TB, this would be three sputum samples, while for suspected non-pulmonary TB infections, the sample type would be determined by disease site (i.e. biopsy samples, pus, early morning urine, etc.) following advice from a microbiologist. Specialist paediatric advice is important for the diagnosis and management of suspected TB in children. If a patient's clinical signs and symptoms are consistent with a diagnosis of active TB, it is important that TB treatment should be started promptly as culture results may take several weeks. Patients with TB should not routinely be admitted to hospital for investigation or treatment unless there is a good clinical or social indication.

The standard UK TB drug treatment regime is based on four drugs. Six months treatment with isoniazid and rifampicin is supplemented in the first two months with pyrazinamide and ethambutol. This treatment would of course be adapted if microbiological evidence of drug resistance is reported. (Note that TB affecting the meninges requires specialist treatment.)

Patients with pulmonary TB may be 'sputum smear positive' at their initial diagnosis. This means that mycobacteria have been seen on microscopic examination of a stained sample of their sputum. This implies that such patients have a high bacterial load in their sputum, indicating that they are more infectious than other patients whose sputum is 'smear negative'. Patients with non-pulmonary TB may also be infectious, for example if they have a TB abscess.

Following initial diagnosis, and while awaiting microbiological confirmation of the diagnosis, all patients should have their risk of drug resistance assessed. Risk factors for TB drug resistance in a patient's history include:

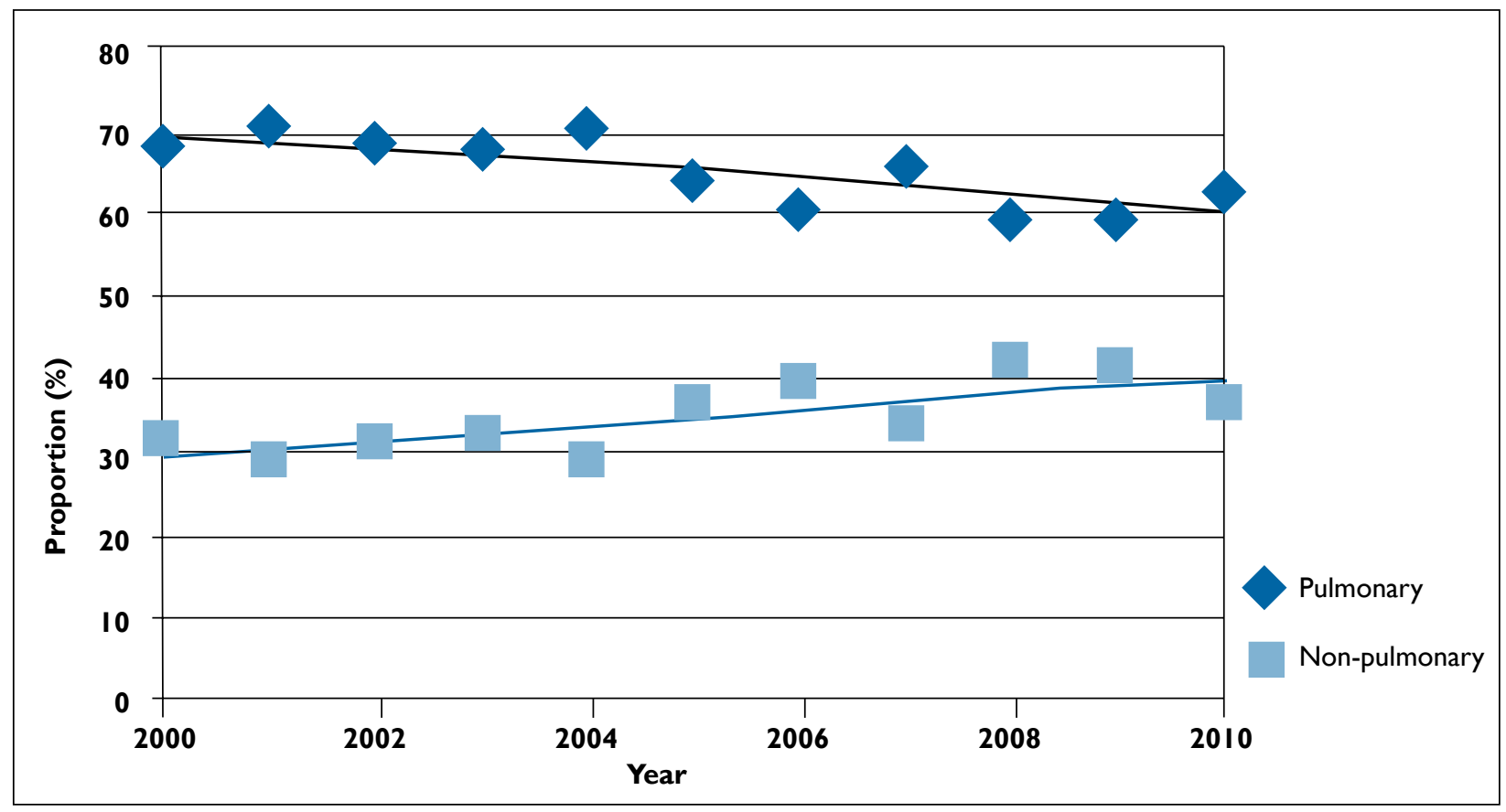

FIGURE 5 Trends in proportions of pulmonary and non-pulmonary tuberculosis cases in Scotland (2000-10). 
- Prior TB drug treatment or prior TB treatment failure

- Contact with a known case of drug-resistant TB

- Birth outside the UK, particularly in a high-incidence country

- HIV co-infection

- Residence in London or another area with a known high TB prevalence

- Age (highest resistance rates found in those aged 25 to 44)

- Sex (highest resistance rates found in men)

If a patient is suspected of being at increased risk of drug-resistant $T B$, this should be discussed with the microbiology service as rapid molecular probes for drug resistance may be appropriate. If a patient is found to have multiple drug-resistant TB (MDR-TB) or extensively drug-resistant TB (XDR-TB), their management should be undertaken by a specialist with experience in MDR-TB.

\section{THE ROLE OF SPECIALIST NURSES}

AllTB patients should be reviewed as early as practicable by a specialist TB nurse. Specialist TB nurses perform a variety of essential functions in the management of $T B$ patients, including:

- Contact tracing

- Supervision of treatment

- Assessment of adherence to treatment

- Monitoring for potential treatment side-effects

- Liaison with patients and the multi-disciplinary care team

Treatment and care of TB patients must take account of their individual needs and preferences. Good communication between healthcare professionals and patients is essential. Specialist TB nurses are ideally placed to build good relationships with their patients to ensure good therapeutic outcomes, providing relevant information appropriate and accessible to each patient's individual needs. The active involvement of a TB nurse in the management of a patient's treatment will enable early identification of problems with adherence to therapy. The TB nurse may recommend that a treatment programme, involving Directly Observed Therapy (DOT) be used where issues with adherence to treatment have been identified. The TB nurses usually initiate contact tracing at a very early stage in their management of a patient with TB. Contact tracing is crucial in preventing TB transmission. It is undertaken both to identify possible sources of a patient's infection (i.e. who they might have acquired it from) as well as identifying secondary cases (i.e. those to whom they may have transmitted the infection). It is often not possible to distinguish between source and secondary cases. Screening for TB should be offered to the household contacts of any person with active TB, irrespective of the site of infection. All household contacts should be screened within six weeks. High-risk contacts (such as children, those who are symptomatic and where the index case has a long history of infection) should be screened more urgently.

Where a TB patient has been found to have sputum smear-positive TB, a more extensive screening may be indicated. The need for this would be informed by consideration of:

- The degree of infectivity of the TB patient

- The length of time the TB patient was in contact with others

- If any contacts are particularly susceptible (i.e. immunocompromised)

- The proximity of contacts

- Casual contacts of TB patients, including most workplace contacts, would not normally be screened

A TB nurse will seek specialist advice about screening contacts of a TB patient where there may have been prolonged close contact in specific settings. These settings have been associated with increased risk of TB transmission and therefore the potential risk of a TB outbreak needs to be considered.These settings include:

- Schools or other educational settings, including nurseries

- Hospital inpatient or care homes

- Residential or corrective institutions

- Workplace settings involving prolonged close contact between workers

- Aircraft flights of greater than eight hours duration

It is important however that the risks in other settings which may be specific to individual TB patients are considered by the TB nurse, consulting with the public health team if appropriate.

\section{TRACING OUTBREAKS}

Recent developments in molecular diagnostic techniques are now being used to establish links between different isolates of M. tuberculosis. The current system being used in the UK is the Mycobacterium Interspersed Repetitive Units - Variable Number of Tandem Repeats (MIRUVNTR). All UK isolates are now typed and the MIRUVNTR data from these are held in a national UK MIRUVNTR database. This enables rapid 'real-time' identification of linked $M$. tuberculosis genotypes for the identification of related cases and outbreaks, so that appropriate public health actions can be initiated. In the 
future, with rapid advances in molecular techniques, it is possible that whole genome sequencing of $M$. tuberculosis will become routine practice. This will enable better understanding of routes of TB transmission, so improving the management of clusters of TB cases.

\section{HIGHLIGHTS}

- The incidence ofTB in the UK is increasing, and non-pulmonary TB is becoming more common.

- Tuberculosis is associated with significant mortality and healthcare cost.

- Underlying patient risk factors are helpful in early assessment of the risks of drug-resistant strains of TB.

- It is essential to screen contacts of a TB case to identify a possible source for their infection, as well as to find and treat possible secondary cases.

- It is important to obtain microbiological samples for confirmation of TB infection and to determine the antibiotic susceptibility of the organism.

- Antibiotic therapy should be guided by culture results, national guidelines and specialist advice.

\section{Acknowledgements}

We thank Eisin MacDonald and Fiona Johnson at HPS for their work on the ESMI data and graphs. We are also grateful to all of the TB clinicians, TB nurses and the public health teams in Scotland for the work they have put into completing ESMI reports. Finally, we thank the staff of Scottish microbiology laboratories including the Scottish Mycobacteria Reference Laboratory, whose work is central to diagnosis and management of TB.

\section{Further reading}

I Abubakar I, Lipman M, Anderson C et al. Tuberculosis in the UK: time to regain control. BMJ 20II; 343:428I. http://dx. doi.org/I 0.I I 36/bmj.d428I

2 Health Protection Agency. Tuberculosis in the UK: 2011 report [Internet]. London: HPA; $201 \mathrm{I}$ [cited 2012 June 6]. Available from: http://www.hpa.org.uk/webc/HPAwebFile/ HPAweb_C/I3I7|3|79|6I2

3 Health Protection Network. Tuberculosis: clinical diagnosis and management of tuberculosis, and measures for its prevention and control in Scotland [Internet]. Glasgow: HPN; 2009 [cited 2012 June 6]. Available from: http://www.documents.hps. scot.nhs.uk/about-hps/hpn/tuberculosis-guidelines.pdf

4 Health Protection Scotland. Enhanced surveillance of mycobacterial infections (ESMI) in Scotland: 2011 tuberculosis annual report for Scotland [Internet]. Glasgow: HPN; 20II [cited 2012 June 6]. Available from: http://www.hps.scot.nhs. uk/ewr/article.aspx

5 National Institute for Clinical Excellence. Clinical diagnosis and management of tuberculosis, and measures for its prevention and control [Internet]. Manchester: NICE; 20II [cited 2012 June 6]. Available from: http://guidance.nice.org. uk/CGII7

6 The Scottish Government. A TB action plan for Scotland [Internet]. Edinburgh:The Scottish Government; 201 I [cited 2012 June 6]. Available from: http://www.scotland.gov.uk/ Resource/Doc/346224/0II5224.pdt 


\section{SELF-ASSESSMENT QUESTIONS}

I. A 26-year-old man from Africa presents with symptoms of tuberculosis (TB). Which ONE of the following is TRUE?

Choose one answer.

A. He is at risk of having a resistant strain of TB.

B. He should be admitted to hospital pending confirmation of the TB diagnosis.

C. He should immediately be commenced on a standard three-drug anti-tuberculous therapy regime.

D. He should not be commenced on treatment until microbiology results, including sensitivities, are available.

E. Notification to public health is not necessary until TB is confirmed.

2. In making a diagnosis of tuberculosis (TB), which ONE of the following is TRUE?

Choose one answer.

A. A patient with sputum smear-positive TB has an increased risk of being infected with a multi-resistant TB strain.

B. A diagnosis cannot be made until there is microbiological confirmation.

C. It is important that at least three samples are sent for microbiology.

D. A patient with non-pulmonary TB is not infectious.

E. A patient with sputum smear-negative pulmonary TB is not infectious.

3. Which ONE of the following is TRUE in Scotland? Choose one answer.

A. The incidence of tuberculosis (TB) amongst children is falling.

B. About one in six of those who are infected with tuberculosis (TB) will die within a year.

C. Healthcare workers have an increased risk of acquiring tuberculosis (TB).

D. Only I5\% of tuberculosis (TB) infections go on to develop active disease, the others recover (although they may have latent TB).

E. Men and women have equal risks of acquiring tuberculosis (TB).
4. A 38-year-old woman who is an injecting drug user and has been diagnosed with sputum smearpositive tuberculosis (TB), is being managed by the TB nursing team. Which ONE of the following is TRUE?

Choose one answer.

A. She should be advised against close contact at home with her partner and her three-year-old child until she has had two weeks TB treatment.

B. Her three-year-old son should not be referred for specialist paediatric opinion unless he has been confirmed to have TB.

C. She should be commenced on directly observed therapy (DOT) as she may have problems with adherence to her TB treatment.

D. She is at risk of having a resistant strain of TB.

E. The TB nurse should consider the TB risks to others around her, including those with whom she may have been using drugs.

5. A 72-year-old woman with sputum smear-positive tuberculosis (TB) who lives alone, is referred to the TB nursing service. Which ONE of the following is TRUE?

Choose one answer.

A. The TB nurse will not need to do any contact tracing as she lives alone.

B. The TB nurse will establish early contact with the patient.

C. It is essential that the source from whom she acquired her TB is identified, so that treatment of the source case can be initiated.

D. The specialist TB nurse will initiate her treatment regime.

E. The role of the TB nurse is to establish details of her contacts for contact tracing.

This paper was originally published as part of the Public Health module in the RCPE Online Continuing Medical Education Programme. Online CME, including the anwers to these questions, is available to Fellows and Members at: http://www.rcpe.ac.uk 